\title{
Bienestar emocional en la vejez avanzada: estudio comparativo por edad y género
}

\author{
Ana Belén Navarro*, Belén Bueno Martínez y José Buz Delgado \\ Universidad de Salamanca
}

(Recibido el 2 de Mayo 2012; Aceptado el 4 de Diciembre 2012)

\begin{abstract}
RESUMEN: En la vejez avanzada, los estados emocionales se convierten en el motivo más importante para mantener la satisfacción con la vida. En este estudio se ha examinado el papel de las emociones positivas y negativas sobre el juicio de satisfacción vital en la vejez avanzada, así como las diferencias de edad y género en una muestra de 400 personas muy mayores de Salamanca, con edades comprendidas entre los 75 y 104 años. Los resultados muestran una mayor frecuencia de emociones positivas, siendo las más frecuentes estar atento, sentirse activo y fuerte y, las menos frecuentes, estar entusiasmado e inspirado. Entre los sentimientos negativos más frecuentes destacan sentirse inquieto, expectante y nervioso, y como menos frecuentes sentirse culpable, hostil y avergonzado. Además, aparecen diferencias de edad (quienes tienen entre 75 y 84 años se sienten más activos, entusiasmados e inspirados) y de género (las mujeres muy mayores se sienten más inquietas, nerviosas, orgullosas, temerosas, asustadas y perturbadas). Por otra parte, el análisis de regresión múltiple pone de manifiesto que permanecer animado, alegre, vigilante y alerta frente a los sucesos resulta funcional en el mantenimiento de la satisfacción vital de las personas de 75 y más años. Estos resultados confirman que las emociones positivas son un recurso potencial para la resiliencia psicológica en la vejez avanzada.
\end{abstract}

Palabras clave: personas muy mayores, resiliencia psicológica, satisfacción vital.

Emotional well-being in advanced old age: comparative study by age and gender

ABSTRACT: In very old age, emotional states become the most important reason to maintain life satisfaction. In this study we examined the role of positive and negative emotions on the judgment of life satisfaction in advanced old age and the age and gender differences in a sample of 400 elderly people of Salamanca, aged between 75 and 104. The results show a higher frequency of positive emotions than negative, with the most frequent of the former being attentive, active and strong, and the less frequent ones being excited and inspired. Among the more frequent negative emotions are feeling jittery, nervous and alert, and the less frequent ones are feeling guilty, hostile and ashamed. In addition, there are differences in terms of both age (people aged between 75 and 84 are more active, enthusiastic and inspired) and gender (very old women are more jittery, nervous, proud, afraid, scared and upset). Moreover, multiple regression analysis 
showed that remaining lively, happy, interested and alert to events is essential for maintaining the life satisfaction of people aged over 75 . These results confirm that positive emotions are a potential resource for psychological resilience in advanced old age.

Keywords: very old people, psychological resilience, life satisfaction.

\section{INTRODUCCIÓN}

El envejecimiento de los muy mayores está suscitando gran interés dentro de la comunidad científica debido a dos razones fundamentales: las personas muy mayores $(75+)$ constituyen el sector de población que más ha aumentado en los últimos años y que más va a crecer en las próximas décadas (ONU, 2002), pero se conoce muy poco específicamente sobre este grupo de edad. En tanto que nueva etapa del ciclo vital, la vejez avanzada plantea numerosos interrogantes a los que algunos investigadores han empezado a responder (v.gr., Berg, Hassing, McClearn, y Johansson, 2006; Jopp y Rott, 2006; Martin, Rott, Poon, Courtenay y Lehr, 2001; Navarro, 2007; Navarro y Bueno, en prensa). Pero a pesar de este mayor interés, desde diferentes frentes se reconoce la importancia de recoger más datos sobre la calidad de vida y la situación funcional de los más mayores en diferentes países y de orientar la investigación y la práctica médica, psiquiátrica, psicológica y sociopolítica hacia esta nueva etapa del ciclo vital.

La cuestión sobre lo que constituye una "buena vida" y sobre cuáles son las condiciones personales y externas que promueven formas deseables de desarrollo personal a lo largo de la vida, tiene especial importancia durante la vejez, etapa en la que las personas mayores han de manejar el balance negativo que supone la mayor presencia de pérdidas que de ganancias (Brandtstädter y Rothermund, 2002).

En los últimos 20 años se ha conseguido un progreso notable en la identificación de los factores que influyen en el bienestar subjetivo (BS). La satisfacción con la vida es uno de los componentes del BS, y refleja, en contraste con el componente afectivo (emociones positivas y negativas), el modo en que las personas evalúan cognitivamente su vida (Diener, Suh, Lucas y Smith, 1999). Diener (1984) definía la satisfacción con la vida como la valoración cognitiva de la propia vida en general, basada en la comparación entre las aspiraciones y los logros conseguidos (Lucas, Diener y Suh, 1996). Las emociones positivas y negativas representan el componente afectivo. Las primeras reflejan la forma en que una persona se siente entusiasmada, activa, alerta, con energía, etc. Por el contrario, las emociones negativas constituyen la dimensión general de distrés subjetivo y conllevan una visión de las obligaciones cotidianas como algo desagradable que empuja a estados emocionales aversivos, tales como disgusto, ira, culpa, miedo y nerviosismo (Diener, 2006).

Aunque en muchos estudios se ha encontrado que los dos componentes del BS están relacionados (Diener, Oishi y Lucas, 2003; Pavot y Diener, 2004), ambos componentes no son idénticos. Si bien Lucas y sus colaboradores (1996) 
demostraron que la satisfacción vital se diferencia claramente tanto del afecto positivo como del afecto negativo, interesa comprender mejor el modo en que se relacionan los componentes afectivos y cognitivos del BS. A pesar de los esfuerzos recientes por comprender dicha interrelación a través de diferentes naciones, poco se sabe sobre dicha interrelación en la vejez avanzada dado que la mayoría de los estudios previos han utilizado muestras de personas jóvenes y adultas (Kuppens, Realo y Diener, 2008; Pavot y Diener, 2004).

La edad y el género son dos variables que permiten comprender mejor la vejez y que pueden ser amortiguadoras o potenciadoras de los efectos negativos del envejecimiento y de su vivencia. Cuando se ha considerado cómo es el BS en función de la edad, se han obtenido resultados variables dependiendo del tipo de medida utilizada y del componente específico del BS sometido a valoración. La mayor parte de los estudios coinciden en que la satisfacción vital es estable a lo largo de la vida (Diener y Suh, 1998), pero los efectos de la edad en el componente afectivo del BS son menos claros y más controvertidos. Mientras algunas investigaciones (v.gr., Charles, Reynolds y Gatz, 2001; Isaacowitz y Smith, 2003; Lawton, 2001) han puesto de manifiesto un efecto significativo de la edad tanto en el afecto positivo como en el negativo, otras han obtenido que personas mayores y jóvenes no se diferenciaban en la frecuencia de emociones negativas, pero sí en la frecuencia de emociones positivas (Mroczek, 2001; Mroczek y Kolark, 1998; Pinquart, 2001). De entre los escasísimos trabajos con personas muy mayores, en el estudio de Berlín (Staudinger, Freund, Linden y Maas, 1999) apareció un modesto declive en el afecto positivo en las personas de 85 y más años, pero no había el correspondiente incremento del afecto negativo. Ferring y Filipp (1995) también señalaron un declive longitudinal del afecto positivo solamente entre las personas muy mayores.

Otra cuestión muy poco investigada tiene que ver con las diferencias en la emocionalidad de las personas muy mayores en función del género. Existe la creencia generalizada de que a lo largo de la juventud y de la vida adulta, las mujeres son más emocionales y más propensas a expresar sus emociones que los hombres (Brody y Hall, 2000; Simon y Nath, 2004). Asimismo, se han encontrado diferencias en la expresión de emociones específicas, en el sentido de que las mujeres expresan con más frecuencia emociones de felicidad, tristeza y miedo, mientras que los hombres expresan más emociones de cólera (Alcalá, Camacho, Giner, Giner e Ibáñez, 2006; Hall y Matsumoto, 2004; Wester, Vogel, Pressly y Heesacker, 2002). ¿Ocurre lo mismo en la vejez avanzada? Hasta la actualidad no se dispone de investigaciones que hayan abordado específicamente las emociones en función de si se es hombre o mujer muy mayor.

Teniendo en cuenta todo lo anterior, el objetivo principal de este estudio fue analizar la relación entre emociones positivas y negativas y juicios de satisfacción vital en personas muy mayores españolas. El presente estudio intenta contestar estos dos interrogantes. Primero, cuáles son las emociones más frecuentes entre las personas muy mayores de nuestro entorno sociocultural y si existen diferencias de edad y de género en la frecuencia de las emociones experimenta- 
das en la vejez avanzada. Segundo, cuál es la contribución de la frecuencia de las emociones positivas y de las emociones negativas sobre los juicios globales de satisfacción vital en personas muy mayores.

En este sentido, planteamos varias hipótesis. En primer lugar y en la línea de Isaacowitz y Smith (2003) y Lyubomirsky, King y Diener (2005), se espera que tanto las emociones positivas como las negativas estén presentes en las personas muy mayores, siendo más frecuentes las emociones positivas. Por lo que respecta a las diferencias de edad y género en la frecuencia de las emociones, y de acuerdo con la mayor parte de la bibliografía, hipotetizamos un mayor declive de las emociones positivas en las personas de más edad (Hilleras, Jorm, Herlitz y Winblad, 1998; Staudinger, Freund, Linden y Maas, 1999) y mayor afecto y emociones negativas en las mujeres (Baltes, Freund y Horgas, 1999; Feingold, 1994; Smith y Baltes, 1999). Por último, se espera que la presencia de emociones positivas y negativas tenga efectos significativos sobre la satisfacción vital en la vejez avanzada (Aspinwall, 2001; Ong, Bergeman, Bisconti y Wallace, 2006; Staudinger, Freund, Linden y Maas, 1999).

\section{MÉTODO}

\section{Participantes}

La muestra que participó en este estudio estuvo compuesta por 400 personas muy mayores (200 hombres y 200 mujeres). Sus edades oscilaban entre los 75 y los 104 años, con una edad media de 85 años (DT=6,38). Las edades de las mujeres iban desde 75 hasta 104 años con una media de 84,93 años y las de los hombres desde 75 hasta 103 años, con una media de 84,76 años. Todos residían en su domicilio en la ciudad de Salamanca. La mayor parte tenía buen estado cognitivo, ausencia de síntomas depresivos y buen estado funcional. Concretamente, un $84,25 \%$ no presentaba deterioro cognitivo y sólo un $15,75 \%$ tenía deterioro cognitivo ligero; el $89,5 \%$ no presentaba depresión y sólo un $10,5 \%$ tenía depresión ligera; y el 53,5\% eran totalmente autónomos, un 43\% presentaba dependencia ligera y tan sólo un 3,5\% tenía dependencia moderada. Sus características sociodemográficas desglosadas pueden verse en la Tabla 1. 
Tabla 1. Datos Sociodemográficos de los Participantes en este Estudio (en la Variable Nivel Educativo Hubo 40 Casos Perdidos -10\%- y en la Variable Nivel Económico Hubo 30 Casos Perdidos -7,5\%-)

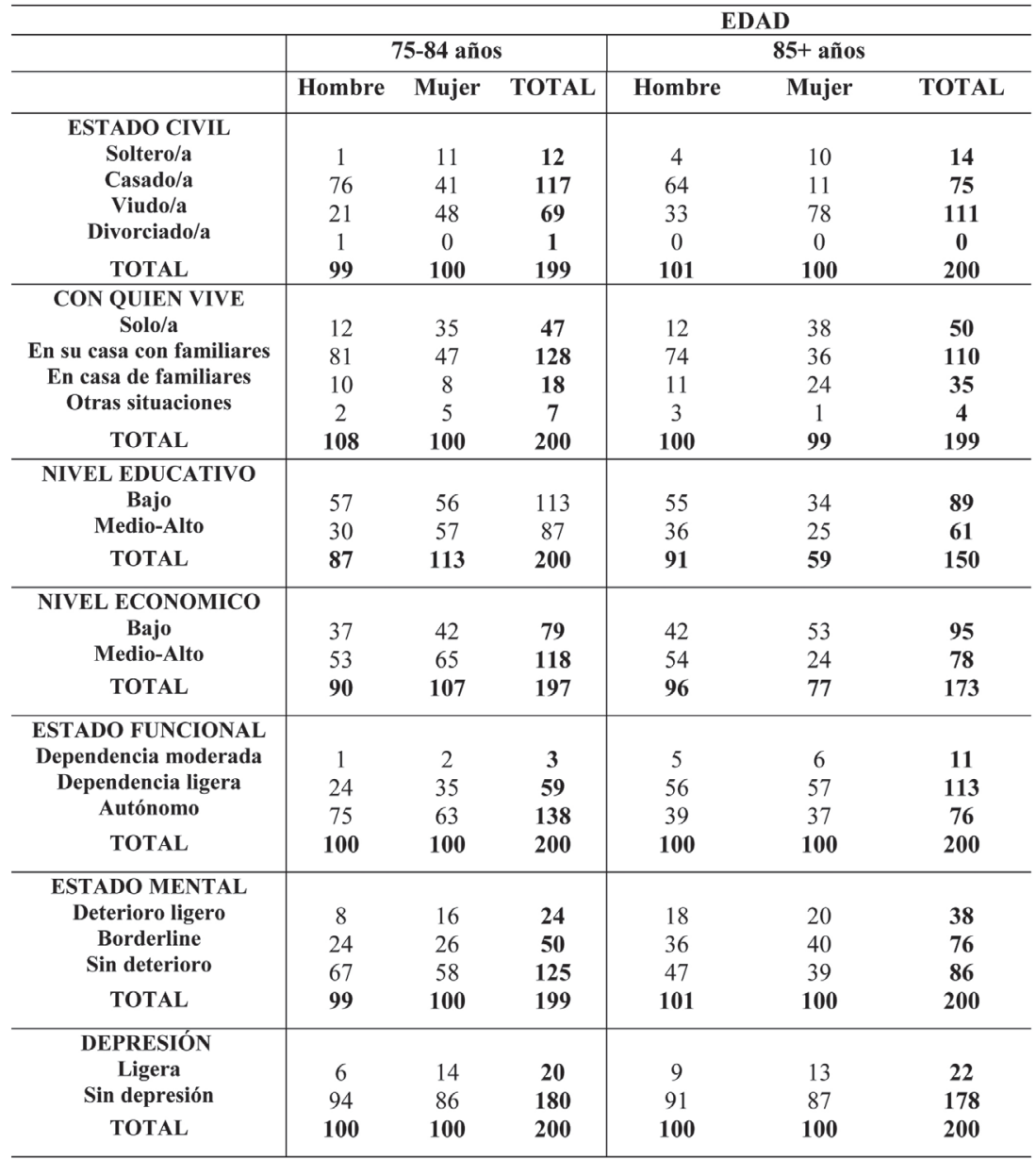

\section{Instrumentos}

Las emociones fueron evaluadas con la versión de la Escala de Afecto Positivo y Negativo -PANAS- (Watson, Clark y Tellegen, 1988), utilizada en el Estudio de Envejecimiento de Berlín -BASE- (Staudinger, Freund, Linden y Maas, 1999). Se seleccionó esta versión por haber sido aplicada en dicho estudio con personas muy mayores y haberse comportado adecuadamente en ese grupo de edad. La versión española del PANAS (Sandín, Chorot, Lostao, Joiner, Santed y Valiente, 1999) se ha validado con jóvenes estudiantes universitarios y la validación previa realizada con mujeres de entre 45 y 65 años de nivel cultural 
medio-bajo (Joiner, Sandín, Chorot, Lostao y Marquina, 1997) no resultó tan óptima como inicialmente se supuso. La Escala de Afecto Positivo y Negativo utilizada se compone de 20 adjetivos que describen distintos sentimientos y emociones (10 para cada dimensión). Mediante opciones de respuesta tipo Likert de 4 puntos se pidió a las personas muy mayores de nuestro estudio que evaluasen su grado de acuerdo o desacuerdo con la presencia de cada uno de los estados de ánimo últimamente. Respetando la estructura de la versión original, se presentó un descriptor simple para cada uno de los 20 estados afectivos, sin reformular ninguno y sin añadir un segundo descriptor a la formulación del afecto en cuestión, a diferencia de las modificaciones introducidas por Sandín et al. (1999). Los índices de consistencia interna (Alpha de Cronbach) obtenidos por nosotros fueron 0,75 para la escala total, 0,84 para la escala de Afecto Positivo y 0,75 para la escala de Afecto Negativo. Mediante análisis factorial exploratorio (Navarro, 2007), se obtuvieron dos factores perfectamente definidos, uno para el afecto positivo (factor 1) y otro relacionado con el afecto negativo (factor 2). Los pesos factoriales son bastante elevados y positivos en el factor relevante y más bajos y negativos en el factor irrelevante. La varianza explicada es muy semejante en ambos factores. Para la muestra total, el Factor 1 explica el 21,11\% y el Factor 2, el 17,86\% de la varianza. Las emociones inquieto/a (alert), orgulloso/a (proud) $y$ expectante (expectant) no pesaron en el factor esperado, lo que indica que esas tres emociones originalmente positivas fueron consideradas por las personas muy mayores de nuestro estudio como negativas.

La satisfacción vital se evaluó con la Escala de Satisfacción con la Vida -SWLS- originalmente desarrollada por Diener, Emmons, Larsen y Griffin (1985). Esta escala, ampliamente utilizada con personas mayores españolas, incluye 5 ítems relacionados con la valoración de la propia vida sobre los que hay que indicar el grado de acuerdo o desacuerdo ("En términos generales, su vida se aproxima a su ideal”; "Las circunstancias de su vida son excelentes"; "Está satisfecho con su vida"; "Hasta ahora ha conseguido las cosas importantes que usted deseaba en la vida"; "Si su vida volviera a empezar, no cambiaría casi nada”). Los 5 ítems forman un único factor. Los participantes respondían utilizando una escala tipo Likert graduada de 1 a 4, desde un total desacuerdo con la frase a un total acuerdo. Por lo tanto, el rango de posibles puntuaciones va desde una mínima satisfacción con la vida (5) a muy alta satisfacción vital (20). La consistencia interna (Alfa de Cronbach) de la escala SWLS en este trabajo fue de 0,71 .

\section{Procedimiento}

La recogida de datos se realizó mediante entrevista individualizada en el domicilio de cada persona mayor. Se seleccionaron encuestadoras a las que se formó para unificar las condiciones de recogida de información y para evitar la inducción de respuestas. Utilizando un muestreo aleatorio de los datos registrados en el censo, se identificaron 400 participantes que cumplieran las condiciones de 
edad y género requeridas. A todos los participantes seleccionados en la primera ronda, se les envió una carta en la que se informaba del estudio y se solicitaba su participación en él. Previendo posibles bajas, se identificaron tres sustitutos con similares características demográficas para cada seleccionado en primera ronda. Se produjo una elevada mortandad experimental por diferentes razones, entre las que figuran ausencia de interés propio en el estudio, fallecimiento de la persona seleccionada, traslado del domicilio sin haber dejado un domicilio alternativo y negativa de los familiares a que sus mayores colaborasen en el estudio. Hubo algunos casos en que la imposibilidad de participar afectó a los tres sustitutos seleccionados inicialmente, por lo que se tuvo que completar la muestra con personas de la misma edad y género, más allá de los sustitutos. En torno a un $25 \%$ de participantes tuvo que ser repuesto. La muestra final con la que se contó para la realización del estudio fue de 400 personas muy mayores.

Transcurridos unos días desde el envío de la carta, las evaluadoras se trasladaban a los domicilios de cada una de las personas mayores, donde se les explicaba el objetivo del estudio y se les aseguraba el anonimato y la confidencialidad de sus respuestas. Tras la obtención del consentimiento informado, se concertaba una cita con ellas en el propio domicilio. En los casos en que la persona mayor se negaba a colaborar, se abandonaba el domicilio y se visitaba al primer sustituto. Si éste también fallaba, se recurría al segundo sustituto y así sucesivamente hasta conseguir un participante que cumpliera los mismos requisitos de edad y género que el seleccionado en primera ronda.

En todo momento se trató de crear una situación natural y un ambiente relajado y cordial para lograr que las respuestas fueran lo más espontáneas y fiables posibles. Se adaptaba el lenguaje utilizado, la petición de información y el tiempo dedicado en función de las características y circunstancias de cada persona mayor.

Toda la fase de recogida de datos duró cuatro meses. Las sesiones de valoración duraban aproximadamente 90 minutos. En algunos casos, hubo que dividir la valoración en varias sesiones. Dado que este trabajo forma parte de un estudio más amplio sobre calidad de vida en personas muy mayores españolas, además de los protocolos incluidos en este trabajo, se aplicaron pruebas de personalidad, afrontamiento, estado cognitivo, afectivo y funcional, calidad de vida relacionada con la salud y actividades sociales y comunitarias.

\section{RESULTADOS}

Todos los análisis estadísticos se realizaron con la versión 19 del programa SPSS para PC, aceptándose un nivel de significación del 95\%.

En las Tablas 2 y 3 se presenta el orden en que las diferentes emociones positivas y negativas son experimentadas por los participantes en este estudio, de acuerdo con sus propias valoraciones, habiéndose realizado diferencias de medias para muestras relacionadas, junto con las medias y las desviaciones típicas de cada una de ellas. 
Tabla 2. Medias y Desviaciones Típicas de las Emociones Positivas Ordenadas por Rangos

\begin{tabular}{ccc}
\hline RANGO & EMOCIONES POSITIVAS & M (DT) \\
\hline 1 & Atento/a & $2,84(0,84)$ \\
2 & Activo/a & $2,82(0,93)$ \\
3 & Fuerte & $2,48(0,97)$ \\
4 & Resuelto/a & $2,45(0,93)$ \\
5 & Motivado/a & $2,40(0,96)$ \\
6 & Entusiasmado/a & $2,38(0,97)$ \\
7 & Inspirado/a & $1,94(0,90)$ \\
\hline
\end{tabular}

Tabla 3. Medias y Desviaciones Típicas de las Emociones Positivas Ordenadas por Rangos

\begin{tabular}{ccc}
\hline RANGO & EMOCIONES NEGATIVAS & M (DT) \\
\hline 1 & Inquieto/a & $1,97(0,96)$ \\
2 & Expectante & $1,90(0,83)$ \\
3 & Nervioso/a & $1,83(0,92)$ \\
4 & Apenado/a & $1,77(0,89)$ \\
& Orgulloso/a & $1,77(0,99)$ \\
5 & Excitado/a & $1,69(0,82)$ \\
6 & Irritable & $1,49(0,76)$ \\
7 & Temeroso/a & $1,39(0,72)$ \\
& Asustado/a & $1,39(0,69)$ \\
9 & Perturbado/a & $1,30(0,64)$ \\
10 & Hostil & $1,21(0,51)$ \\
11 & Culpable & $1,12(0,40)$ \\
\hline
\end{tabular}

Como puede observarse, las emociones positivas, indicadoras de participación activa y conexión con el entorno, tales como atento/a, activo/a, fuerte y resuelto/a, son las más experimentadas por las personas muy mayores (Tabla 2). Emociones como motivado/a y entusiasmado/a son también bastante frecuentes. Esto significa que las personas muy mayores de este estudio en algunos momentos sienten admiración e interés por las cosas. Por el contrario, la emoción positiva inspirado/a es la menos frecuente; los muy mayores no se sienten con ideas creativas y novedosas. 
En relación con las emociones negativas, las personas muy mayores se caracterizan por sentirse inquietas, expectantes, nerviosas, apenadas y orgullosas (Tabla 3). Además, las emociones excitado/a, irritable, temeroso/a, asustado/a y perturbado/a ocupan posiciones intermedias, es decir, los muy mayores experimentan con bastante frecuencia sentimientos de estimulación o activación, irritabilidad, miedo e intranquilidad. Sin embargo, las emociones negativas avergonzado/a, culpable y hostil ocupan los últimos lugares, lo que significa que las personas mayores de 75 años apenas sienten vergüenza o culpa, ni muestran sentimientos de frustración y rencor.

En cuanto a las diferencias de edad y de género en las emociones positivas y negativas, únicamente se obtuvieron diferencias de edad estadísticamente significativas en las emociones positivas activo/a, entusiasmado/a e inspirado/a. Sin embargo, no aparecieron diferencias de género en dichas emociones (Tabla 4).

Tabla 4. Medias y Desviaciones Típicas (entre paréntesis) de las Emociones Positivas en función de la Edad y del Género junto con las Diferencias Significativas y no Significativas con sus Valores $F$

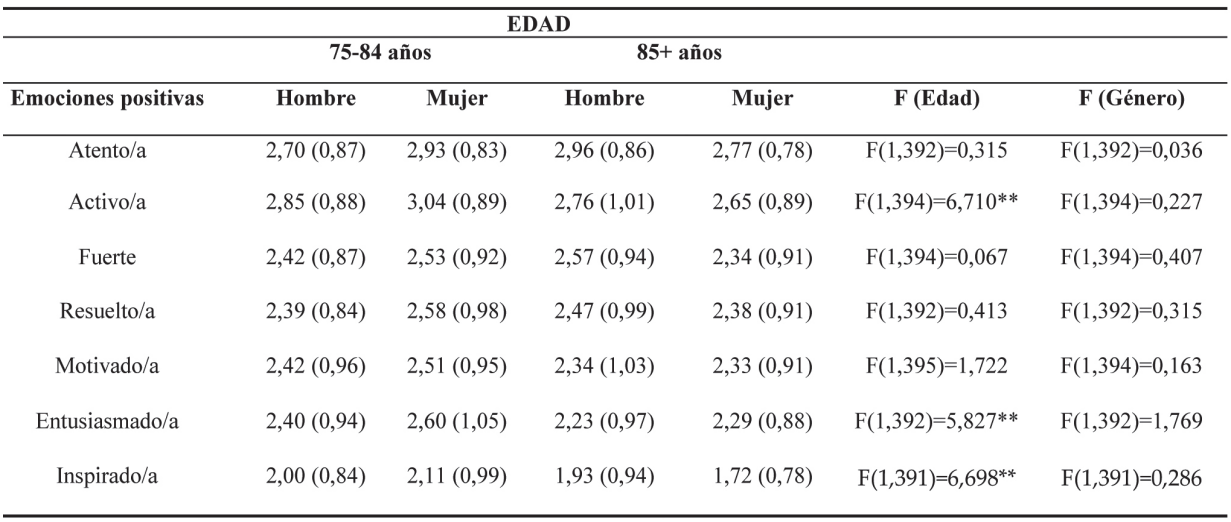

${ }^{*} \mathrm{p}=0,05 ; * * \mathrm{p}=0,01$

Asimismo, aparecieron diferencias de género y no de edad en el afecto negativo y en las emociones negativas: inquieto/a, nervioso/a, orgulloso/a, temeroso/a, asustado/a, perturbado/a y avergonzado/a (Tabla 5). 
Tabla 5. Medias y Desviaciones Típicas (entre paréntesis) de las Emociones Negativas en función de la Edad y del Género junto con las Diferencias Significativas y no Significativas con sus Valores $F$

\begin{tabular}{|c|c|c|c|c|c|c|}
\hline \multirow[b]{3}{*}{ Emociones negativas } & \multicolumn{4}{|c|}{ EDAD } & \multirow[b]{3}{*}{ F (Edad) } & \multirow[b]{3}{*}{ F (Género) } \\
\hline & \multicolumn{2}{|c|}{ 75-84 años } & \multicolumn{2}{|c|}{$85+$ años } & & \\
\hline & Hombre & Mujer & Hombre & Mujer & & \\
\hline Inquieto/a & $1,83(0,89)$ & $2,16(0,99)$ & $1,90(1,03)$ & $2,00(0,90)$ & $\mathrm{F}(1,392)=0,233$ & $\mathrm{~F}(1,392)=5,174^{*}$ \\
\hline Expectante & $1,80(0,81)$ & $1,97(0,84)$ & $1,99(0,88)$ & $1,82(0,78)$ & $F(1,393)=0,048$ & $F(1,393)=0,001$ \\
\hline Nervioso/a & $1,78(0,81)$ & $2,01(1,00)$ & $1,62(0,92)$ & $1,91(0,91)$ & $F(1,393)=1,996$ & $\mathrm{~F}(1,393)=7,734 * *$ \\
\hline Apenado/a & $1,65(0,87)$ & $1,90(0,86)$ & $1,60(0,85)$ & $1,93(0,95)$ & $F(1,395)=0,020$ & $\mathrm{~F}(1,395)=1,052$ \\
\hline Orgulloso/a & $1,63(0,87)$ & $1,93(1,06)$ & $1,58(0,94)$ & $1,93(1,05)$ & $F(1,394)=0,075$ & $\mathrm{~F}(1,394)=10,603^{* *}$ \\
\hline Excitado/a & $1,60(0,75)$ & $1,64(0,82)$ & $1,81(0,86)$ & $1,72(0,83)$ & $F(1,395)=0,098$ & $F(1,395)=3,028$ \\
\hline Irritable & $1,42(0,68)$ & $1,49(0,73)$ & $1,56(0,87)$ & $1,50(0,74)$ & $F(1,395)=0,921$ & $\mathrm{~F}(1,395)=0,011$ \\
\hline Temeroso/a & $1,32(0,70)$ & $1,52(0,81)$ & $1,22(0,56)$ & $1,50(0,77)$ & $F(1,383)=0,630$ & $\mathrm{~F}(1,383)=10,585^{* *}$ \\
\hline Asustado/a & $1,31(0,59)$ & $1,54(0,80)$ & $1,23(0,55)$ & $1,47(0,75)$ & $\mathrm{F}(1,394)=1,024$ & $\mathrm{~F}(1,394)=11,443 * *$ \\
\hline Perturbado/a & $1,23(0,58)$ & $1,35(0,66)$ & $1,22(0,52)$ & $1,42(0,76)$ & $F(1,392)=0,234$ & $\mathrm{~F}(1,392)=6,221^{*}$ \\
\hline Hostil & $1,20(0,55)$ & $1,23(1,51)$ & $1,16(0,50)$ & $1,23(0,48)$ & $F(1,393)=0,159$ & $\mathrm{~F}(1,393)=0,802$ \\
\hline Culpable & $1,04(0,19)$ & $1,17(0,53)$ & $1,14(0,37)$ & $1,12(0,40)$ & $F(1,393)=0,450$ & $F(1,393)=1,687$ \\
\hline Avergonzado/a & $1,09(0,32)$ & $1,08(0,31)$ & $1,15(0,52)$ & $1,02(0,14)$ & $F(1,394)=0,002$ & $\mathrm{~F}(1,394)=3,954^{*}$ \\
\hline
\end{tabular}

$513 * \mathrm{p}=0,05 ; * \mathrm{p}=0,01$

Las mujeres muy mayores se caracterizan por experimentar más afecto negativo, sintiéndose más inquietas, nerviosas, orgullosas, temerosas, asustadas y perturbadas que los hombres muy mayores. Éstos sólo se sienten más avergonzados que las mujeres muy mayores.

Para conocer la contribución de las emociones sobre los juicios de satisfacción vital durante la vejez avanzada, se realizaron análisis de regresión múltiple simultánea. Para ello, se tomó como criterio la satisfacción vital y, siguiendo la recomendación de Hosmer y Lemeshow (1989), se incluyeron como variables predictoras las que habían obtenido una correlación significativa con un nivel de confianza superior o igual a 0,95. Esto hizo que se incluyeran como variables independientes, el afecto positivo y las emociones motivado/a, fuerte, entusiasmado/a, inspirado/a, resuelto/a, atento/a y activo/a, el afecto negativo y las emociones apenado/a, excitado/a, perturbado/a, culpable, asustado/a, hostil, irritable, nervioso/a y temeroso/a.

Se obtuvo que únicamente dos variables (las emociones "atento/a" y "apenado/a") explican un $22,9 \%$ de la varianza en la satisfacción con la vida, tal como indica la $\mathrm{R}^{2}$ corregida $\left(\mathrm{F}_{18,370}=5,809 ; \mathrm{p} \leq 0,001\right)$.

De acuerdo con los coeficientes estandarizados, ambas emociones atento/a y 
apenado/a tienen cargas explicativas similares pero con diferente signo, siendo positiva para la emoción atento/a $(\beta=0,589, \mathrm{p}=0,015)$ y negativa para apenado/a $(\beta=-0,587, p=0,018)$. Esto significa que cuanto más animadas, energéticas, alegres y atentas sean las personas muy mayores y menos tristes y apenadas, mayor será su nivel de satisfacción en esta etapa de la vida.

\section{DISCUSIÓN}

Este trabajo presenta un análisis del papel de la edad y del género en la frecuencia de emociones positivas y negativas en personas muy mayores españolas, así como del modo en que dichas emociones contribuyen sobre la satisfacción vital en la vejez avanzada.

De acuerdo con los resultados obtenidos, las personas muy mayores experimentan más afecto y emociones positivas que negativas, pero a mayor edad, disminuye la frecuencia de emociones positivas; las mujeres muy mayores muestran más emociones negativas que los hombres muy mayores y, por último, las emociones son relevantes como promotoras de la satisfacción vital en la vejez avanzada. Teniendo en cuenta estos hallazgos, creemos que el estudio de los factores sociodemográficos es relevante dentro de un modelo general de Resiliencia psicológica, puesto que se asocian y funcionan como moderadores de los efectos negativos del envejecimiento y de su vivencia.

La frecuencia de afecto positivo y negativo en las personas muy mayores de este estudio es similar a la encontrada en algunos trabajos anteriores (v.gr., Staudinger, Freund, Linden y Maas, 1999), donde también figuraban como sentimientos positivos más frecuentes estar atento/a y sentirse activo/a y fuerte $\mathrm{y}$, como menos frecuentes, estar entusiasmado/a e inspirado/a. Entre los sentimientos negativos mencionados con más frecuencia figuran sentirse inquieto/a, expectante y nervioso/a, y como menos frecuentes sentirse culpable, hostil y avergonzado/a.

Es necesario detenerse en el hallazgo de que aunque la estructura absoluta del afecto positivo y del afecto negativo parece relativamente invariable en diferentes culturas, las cargas de algunos términos individuales pueden verse afectados por cuestiones lingüísticas y culturales. De modo similar a lo ocurrido en varios estudios previos con algunos términos individuales (v.gr., Joiner et al. 1997; Watson et al. 1984), en este trabajo ha habido tres adjetivos que han sido interpretados por los participantes con valencia afectiva diferente a la inicialmente hipotetizada. Este ha sido el caso de los estados afectivos inquieto/a (alert), orgulloso/a (proud) y expectante (expectant). Probablemente esto se haya debido al doble significado y/o falta de pureza de estas palabras. Por ejemplo, la palabra orgulloso/a en español tiene el doble significado de orgullo y vanagloria, y también puede haber una actitud cultural entre las personas más mayores españolas tendente a presentaciones asertivas del orgullo. Las emociones inquieto/a y expectante pueden resultar un tanto ambiguas, dependiendo de si su significado se centra en la parte estimulante de ambas o en la parte que puede denotar ner- 
viosismo. Al tratarse de estados afectivos mixtos, los resultados indican que las personas muy mayores estudiadas han interpretado estos términos en su vertiente afectiva negativa. Convendría tener en cuenta estos hallazgos para, en futuros trabajos, intentar elegir términos que recojan estados afectivos puros, tal como fue inicialmente la idea original de los creadores de la escala PANAS y como ha sido defendido por Sandín et al (1999).

Por otra parte, dados los cambios negativos asociados al hacerse muy mayor que se producen en condiciones de vida objetivas tales como ingresos, salud, apoyo social, etc., sería lógico esperar una mayor prevalencia de afecto y emociones negativas. Sin embargo, sucede lo contrario, se experimentan con más frecuencia emociones positivas que negativas. Esto es interpretado como un signo de Resiliencia psicológica (Staudinger et al., 1999), lo que podría ser debido al hecho de que, tal como defienden Gross, Carstensen, Pasupathi, Tsai, Skorpen y Hsu (1997), para influir en sus emociones las personas muy mayores adoptan en mayor medida estrategias centradas en los antecedentes, es decir, alteran el curso de las emociones antes de que la emoción comience a experimentarse, cambiando el ambiente o cambiando el significado de la situación. Esto conduciría a reducir de una manera efectiva la experiencia subjetiva de la emoción negativa, lo que tiene consecuencias adaptativas para la persona muy mayor.

En este estudio se ha encontrado que la edad muy avanzada influye únicamente en las emociones positivas. Las personas de 85 y más años manifiestan sentirse menos activas, entusiasmadas e inspiradas que las de menor edad (entre 75 y 84 años). Este resultado puede interpretarse como un signo de reducción de los recursos psicológicos que se produce con la edad, que se hace más relevante en las últimas etapas de la vida. Resultados similares fueron encontrados en estudios transversales previos (Lawton, 2001; Mroczek y Kolarz, 1998; Stacey y Gatz, 1991). En ellos, jóvenes y mayores no se diferenciaban en la frecuencia de emociones negativas, pero sí en la de las emociones positivas. A lo largo de la vida adulta, las personas cuentan con muchas experiencias emocionales que pueden hacer que el umbral para las respuestas emocionales aumente con la edad. Sin embargo, en la vejez avanzada esas experiencias se reducen, lo que puede afectar a la frecuencia de las emociones que se experimentan. Esta disminución puede estar asociada con el incremento de las pérdidas en la red social y con la reducción del tiempo que se emplea en actividades sociales, lo cual proporcionaría un contexto adecuado para expresarse emocionalmente de un modo más positivo (Hilleras, Jorm, Herlitz y Winblad, 1998). Nuestro estudio pone de manifiesto que esto es lo que pasa en la vejez avanzada, lo que ayuda a completar el conocimiento sobre los cambios emocionales a lo largo de todo el ciclo vital, hasta edades muy avanzadas (incluyendo octogenarios, nonagenarios y centenarios).

Respecto a las diferencias de género, en este estudio se ha encontrado que ser hombre y mujer muy mayor también influye en la frecuencia de algunas emociones negativas, pero en ninguna de las positivas. Las mujeres muy mayores se sienten en mayor medida inquietas, nerviosas, temerosas, asustadas y perturba- 
das. Este hecho resulta consistente con los estudios epidemiológicos que muestran cómo las mujeres presentan frecuentemente síntomas asociados al estrés, la ansiedad o la depresión (Beekman et al., 1998; Imserso, 2005; Simon y Nath, 2004). Todos estos estudios han encontrado que, aunque en la vejez aparece una menor tasa de estos trastornos en comparación con otras etapas de la vida, la mujer sigue presentando mayores niveles de ansiedad que los hombres (10-12\% frente al 5-6\%) y de sintomatología depresiva (13,7\% frente al 5,8\%). Nuestro trabajo aporta evidencia de que esta situación diferencial por género también se produce a edades muy avanzadas.

Considerando que la salud no sólo es la ausencia de enfermedad y que las emociones positivas son algo más que la ausencia de emociones negativas, es esperable que las emociones positivas sean útiles para prevenir enfermedades, reducir su intensidad y duración y, también, para alcanzar niveles elevados de satisfacción con la vida (Lyubomirsky, King y Diener, 2005). Tras la realización de este estudio, se puede afirmar que permanecer vigilante y alerta frente a los sucesos resulta funcional en el mantenimiento de la satisfacción vital de las personas de más de 75 años. Por lo tanto, dicha emoción resulta protectora frente al estrés que acompaña a las diferentes pérdidas propias de esta etapa de la vida.

Junto a la emoción positiva "atento/a" aparece una emoción negativa, "apenado/a". Dicha emoción puede ser considerada un factor importante de riesgo en las personas muy mayores. Cuanto más desanimada y triste esté una persona a estas edades, menor será su satisfacción con la vida. Este resultado es congruente con la mayor parte de los estudios que han tratado de relacionar las emociones negativas con la salud y con la satisfacción (Blazer, 2003; Hawkley, Masi, Berry y Cacioppo, 2006). En ellos se muestran los efectos adversos de las emociones negativas sobre la salud de las personas mayores, manifestados en forma de enfermedades cardiovasculares, conductas poco saludables, disconformidad con las prescripciones médicas, dificultades en la rehabilitación, etc. Nuestro trabajo supone una aportación novedosa en relación con el papel de algunas emociones sobre una dimensión importante del bienestar subjetivo y de la calidad de vida en las personas muy mayores de nuestro entorno sociocultural.

Es preciso señalar que la naturaleza transversal de este estudio es una limitación importante que puede haber dificultado la interpretación de las diferencias de edad encontradas, debido a la posible confusión entre los factores asociados a la edad y los efectos generacionales.

La supervivencia selectiva es posible que también haya intervenido en la dirección de los resultados: las escasas diferencias relacionadas con la edad y con el género pueden estar reflejando que las emociones en la vejez avanzada sean simplemente un efecto de selección consistente en que los más resistentes es más probable que vivan más años. Además, que las relaciones entre la satisfacción con la vida y las emociones se hayan estudiado sin considerar determinados factores de riesgo como pueden ser las condiciones de salud objetivas, probablemente sea otra limitación. En próximos trabajos se recogerán datos complementarios que ayuden a matizar esta cuestión. 
Asimismo, es probable que las personas muy mayores no hayan comprendido bien algunas emociones, quizás por el carácter ambivalente de algunas o incluso debido al cansancio que supuso la aplicación completa de los instrumentos.

En todo caso, a pesar de las limitaciones, los hallazgos de este trabajo apoyan la relevancia de las emociones en la vejez avanzada, lo que supone un progreso en el conocimiento de esta etapa de la vida que ha estado olvidada en gran parte de los trabajos de investigación hasta ahora.

\section{REFERENCIAS}

Alcalá, V., Camacho, M., Giner, D., Giner, J. e Ibáñez, E. (2006). Afectos y género. Psicothema, 18(1), 143-148.

Aspinwall, L. G. (2001). Dealing with adversity: Self-regulation, coping, adaptation, and health. En A. Tesser, y N. Schwarz (Eds.), The Blackwell Handbook of Social Psychology: Vol. 1. Intrapersonal Processes (pp. 591-614). Malden, MA: Blackwell.

Baltes, P. B., Freund, A. M. y Horgas, A. L. (1999). Men and women in the Berlin Aging Study. En P. B. Baltes, y K. U. Mayer (Eds.), The Berlin Aging Study: Aging from 70 to 100 (pp. 259-281). Nueva York: Cambridge University Press.

Beekman, A., Bremmer, M., Deeg, D., Van Balkom, A. J., Smit, J. H., de Beurs, E., Van Dyck, R. y Van Tilburg, W. (1998). Anxiety disorders in later life. A report from Longitudinal Aging Study Amsterdam. International Journal of Geriatric Psychiatric, 13, 717-726.

Berg, A. I., Hassing, L. B., McClearn, G. E. y Johansson, B. (2006). What matters for life satisfaction in the oldest-old? Aging and Mental Health, 10(3), 257-264.

Blazer, D. G. (2003). Depression in late life: Review and commentary. Journals of Gerontology: Series A: Biological Sciences y Medical Sciences, 58A (3), 249-265.

Brandtstädter, J. y Rothermund, K. (2002). The life-course dynamics of goal pursuit and goal adjustment: A two-process framework. Developmental Review, 22, 117-150.

Brody, L y Hall, J. (2000). Gender, emotion, and expression. En M. Lewis y J. Haviland-Jones (Eds.), Handbook of emotions (pp. 338-349). Nueva York: The Guilford Press.

Charles, S. T., Reynolds, C. y Gatz, M. (2001). Age-related differences and change in positive and negative affect over 23 years. Journal of Personality and Social Psychology, 80, 136-151.

Diener, E. (1984). Subjective well-being. Psychological Bulletin, 95(3), 542575.

Diener, E. (2006). Guidelines for national indicators of subjective well-being and ill-being. Applied Research in Quality of Life, 1, 151-157.

Diener, E., Emmons, R. A., Larsen, R. J. y Griffin, S. (1985). The Satisfaction 
with Life Scale. Journal of Personality Assessment, 49, 71-75.

Diener, E., Oishi, S. y Lucas, R. E. (2003). Personality, culture, and subjective well-being: Emotional and cognitive evaluations of life. Annual Review of Psychology, 54, 403-425.

Diener, E. y Suh, E. (1998). Age and subjective well-being: An international analysis. Annual Review of Gerontology and Geriatrics, 17, 304-324.

Diener, E., Suh, E. M., Lucas, R. E. y Smith, H. L. (1999). Subjective well-being: Three decades of progress. Psychological Bulletin, 125(2), 276-302.

Feingold, A. (1994). Gender differences in personality: A meta-analysis. Psychological Bulletin, 116, 429-456.

Ferring, D. y Filipp, S. H. (1995). The structure of subjective well-being in the elderly: A test of different models by structural equation modeling. European Journal of Psychological Assessment, 11, 32.

Gross, J., Carstensen, L. L., Pasupathi, M., Tsai, J., GötestamSkorpen, C. y Hsu, A. (1997). Emotion and aging: Experience, expression and control. Psychology and Aging, 12, 590-599.

Hall, J. A. y Matsumoto, D. (2004). Sex differences in judgments of multiple emotions from facial expressions. Emotion, 4, 201-206.

Hawkley, L. C., Masi, C., Berry, J. D. y Cacioppo, J. T. (2006). Loneliness is a unique predictor of age-related differences in systolic blood pressure. Psychology and Aging, 21(1), 152-164.

Hilleras, P. K., Jorm, A. F., Herlitz, A. y Winblad, B. (1998). Negative and positive affect among the very old: A survey on a sample age 90 years or older. Research on Aging, 20, 593-610.

Hosmer, D. W. y Lemeshow, S. (1989). Applied Logistic Regression. Nueva York: John Wiley \& Sons.

IMSERSO (2005). Las personas mayores en España. Informe 2004. Madrid: Ministerio de Trabajo y Asuntos Sociales.

Isaacowitz, D. M. y Smith, J. (2003). Positive and negative affect in very old age. Journal of Gerontology: Psychological Sciences, 58B(3), P143-P152.

Joiner, T. E., Sandín, B., Chorot, P., Lostao, L. y Marquina, G. (1997). Development and factor analytic validation of the SPANAS among women in Spain: (More) Cross-cultural convergence in the structure of mood. Journal of Personality Assessment, 68(3), 600-615.

Jopp, D. y Rott, C. (2006). Adapting to very old age: Exploring the role of personal resources and protective mechanism in centenarians. Psychology and Aging, 21, 266-280.

Kuppens, P., Realo, A. y Diener, E. (2008). The role of positive and negative emotions in life satisfaction judgment across nations. Journal of Personality and Social Psychology, 95(1), 66-75.

Lawton, M. P. (2001). Emotion in later life. Current Directions in Psychological Science, 10(4), 120-123.

Lucas, R. E., Diener, E. y Suh, E. (1996). Discriminant validity of well-being measures. Journal of Personality and Social Psychology, 71, 616-628. 
Lyubomirsky, S., King, L. y Diener, E. (2005). The benefits of frequent positive affect: Does happiness lead to success? Psychological Bulletin, 131(6), 803855.

Martin, P., Rott, C., Poon, L. W., Courtenay, B. y Lehr, U. (2001). A molecular view of coping behavior in older adults. Journal of Aging and Health, 13, 72-91.

Mroczek, D. K. (2001). Age and emotion in adulthood. Current Directions in Psychological Science, 10(3), 87-90.

Mroczek, D. K. y Kolarz, C. M. (1998). The effect of age on positive and negative affect. Journal of Personality and Social Psychology, 75, 1333-1349.

Navarro, A. B. (2007). Rasgos de personalidad, emociones y experiencia temporal: Contribución sobre la Resistencia Psicológica en la vejez avanzada. Tesis Doctoral no publicada, Facultad de Psicología, Universidad de Salamanca, Salamanca, España.

Navarro, A. B. y Bueno, B. (en prensa). Afrontamiento de problemas de salud en personas muy mayores. International Journal of Clinical and Health Psychology.

Ong, A. D., Bergeman, C. S., Bisconti, T. L. y Wallace, K. A. (2006). Psychological resilience, positive emotions, and successful adaptation to stress in later life. Journal of Personality and Social Psychology, 91, 730-749.

ONU (2002). Plan de Acción Internacional de Madrid sobre el envejecimiento 2002. Revista Española de Geriatría y Gerontología, 37 (S2), 12-36.

Pavot, W. y Diener, E. (2004). The subjective evaluation of well-being in adulthood: Findings and implications. Ageing International, 29 (2), 113-135.

Pinquart, M. (2001). Correlates of subjective health in older adults: A meta-analysis. Psychology and Aging, 16, 414-426.

Sandín, B., Chorot, P., Lostao, L., Joiner, T. E., Santed, M. A. y Valiente, R. M. (1999). Escalas PANAS de afecto positivo y negativo: Validación factorial y convergencia transcultural. Psicothema, 11 (1), 37-51.

Simon, R. W. y Nath, L. E. (2004). Gender and emotion in the United States: Do men and women differ in self-reports of feelings and expressive behaviour? The American Journal of Sociology, 109(5), 1137-1177.

Smith, J. y Baltes, P. B. (1999). Trends and profiles of psychological functioning in very old age. En P. B. Baltes y K. U. Mayer (Eds.), The Berlin Aging Study: Aging from 70 to 100 (pp. 197-226). Nueva York: Cambridge University Press.

Stacey, C. A. y Gatz, M. (1991). Cross-sectional age differences and longitudinal change on the Bradburn Affect Balance Scale. Journals of Gerontology: Psychological Sciences, 46, 76-78.

Staudinger, U. M., Freund, A. M., Linden, M. y Maas, I. (1999). Self, personality, and life regulation: Facets of psychological resilience in old age. En P. B. Baltes y K. U. Mayer (Eds.), The Berlin aging study: Aging from 70 to 100 (pp. 302-328). Nueva York: Cambridge University Press.

Watson, D., Clark, L. A. y Tellegen, A. (1984). Cross-cultural convergence in the 
structure of mood: A Japanese replication and a comparison with U. S. findings. Journal of Personality and Social Psychology, 47, 127-144.

Watson, D., Clark, L. A. y Tellegen, A. (1988). Development and validation of brief measures of positive and negative affect: The PANAS scales. Journal of Personality and Social Psychology, 54, 1063-1070.

Wester, S. R., Vogel, D. L., Pressly, P. K. y Heesacker, M. (2002). Sex difference in emotion: A critical review of the literature and implications for counseling psychology. The Counseling Psychologist, 39 (4), 630-652. 